\title{
Association of antenatal care and place of delivery with newborn care practices: evidence from a cross-sectional survey in rural Uttar Pradesh, India
}

Niveditha Devasenapathy ${ }^{1 *}$, Sutapa B. Neogi ${ }^{1}$, Srinivasan Soundararajan², Danish Ahmad², Avishek Hazra ${ }^{3}$, Jaleel Ahmad ${ }^{3}$, Neelakshi Mann ${ }^{4}$ and Dileep Mavalankar ${ }^{5}$

\begin{abstract}
Background: Appropriate immediate newborn care is vital for neonatal survival. Antenatal period is a crucial time to impart knowledge and awareness to mothers regarding newborn care, either during facility visits or during home visits by community health workers (CHWs) especially in the rural context. In this paper, we report newborn care practices in rural Uttar Pradesh (UP) and have explored association between newborn care practices with antenatal care, contact with community health workers during pregnancy and place of childbirth.

Methods: We use cross-sectional baseline data (which is part of a larger intervention project) collected from 129 gram panchayats (GPs) from 15 administrative blocks spread over five districts of UP in 2013. From currently married women $(n=2208)$ of $15-49$ years, who delivered 15 months prior to the survey, we collected information on women's demographic and socio-economic characteristics, knowledge and practice of reproductive, maternal, newborn, child health and nutrition behaviours. Association of newborn practices with antenatal care, contacts by community health worker during pregnancy and place of childbirth were tested using random intercept logistic regression, adjusting for socio-economic and demographic factors and accounting for clustering at the GP and block levels.
\end{abstract}

Results: Eighty-three percent of 2208 mothers received ANC, but only half of the respondents received a minimum of three ANC visits. More than two thirds of respondents delivered at a health facility. Practice of newborn care was poor: merely one fourth of women practised clean cord care, one third of women followed good breastfeeding practices (initiation with an hour of birth, fed colostrum and did not give pre-lacteal feeds) and one third provided adequate thermal care (kept baby warm and delayed bathing). Only 5\% followed all above practices with evidence of clustering of newborn care practices at the block and GP levels. While facility-based childbirth was strongly associated with appropriate newborn care practices, ANC visits and contacts with CHWs was not associated with all newborn care practices.

Conclusion: The quality of ANC care provided needs to be improved to have an impact on newborn care practices. Our finding emphasizes the importance of facility-based birthing. There is a need for training CHWs to strengthen their counselling skills on newborn care. Variation of newborn care practices between communities should be taken into consideration while implementing any intervention to optimize benefits.

Keywords: Newborn care, Antenatal care, Delivery, Community health workers

\footnotetext{
* Correspondence: niveditha@iiphd.org

${ }^{1}$ Indian Institute of Public Health-Delhi, Plot No. 47, Sector 44, Institutional

Area, Gurgaon 122002, India

Full list of author information is available at the end of the article
} 


\section{Key messages}

- Only $5 \%$ of mothers practised all three newborn care practices, namely timely initiation of breast feeding, thermal care and clean cord care.

- Facility-based deliveries are associated with better newborn care practices as compared to home delivery.

- There is a need to emphasize on the quality of counselling during antenatal care (ANC) visits and home visits by community health workers to increase adoption of preventive newborn care practices by newly delivered mothers.

- Significant heterogeneity in newborn practices across blocks and smaller units (gram panchayats) exists. Need to identify cluster-level factors for better implementation of programmes and interventions.

\section{Background}

Major causes for neonatal deaths are due to preterm births and intrapartum complication [1]. While adequate antenatal care and delivery at health facility have shown to reduce stillbirths and is vital for safe mother-newborn dyad; clean cord care, thermal care and appropriate timely initiation of breast-feeding practices are important contributing factors for reduction in newborn morbidity and mortality [2, 3]. As per WHO guidelines, initiation of breast feeding soon after birth, prevention of hypothermia and infections and clean cord care are recommended for all newborns. Use of chlorhexidine may be considered only to replace application of harmful substances, such as cow dung to the cord stump [4]. Antenatal period is the ideal time to impart knowledge regarding appropriate immediate newborn care practices when the women visit hospitals and during home visits made by community health workers. However, studies have shown that in the context of developing countries, antenatal care interventions or completing adequate number of ANC visits may not be associated with lower risk of neonatal death $[5,6]$. This could be due to a gap between coverage and quality of ANC. Delivery at hospitals and a good intrapartum care have shown to have impact on newborn care practices. A nationally representative study from India has shown that facility-based delivery followed by adequate postnatal check-ups substantially reduced neonatal deaths as compared to mothers that had just facility delivery without any postnatal check-up [7] emphasizing the importance of postnatal care in the early newborn period. Another study by Khan et al. [8] in Uttar Pradesh showed that the financial incentives for delivering at a health facility increased client-provider contact, uptake of minimum of three ANC visits and facility-based delivery. These practices together provide windows of opportunity for providing counselling and advice which, in turn, trigger the adoption of a cluster of healthy behaviours that have a direct bearing on maternal and child health.

There is a decreasing trend in neonatal mortality across Indian states. As per Sample Registration System (SRS), the neonatal mortality rate (NMR) (2012) for Uttar Pradesh is 39 per 1000 live births which contributes to $27 \%$ of India's total NMR burden $[9,10]$. Among the rural population, $34 \%$ receive at least three ANC visits, $60 \%$ of women give birth at a health facility and only one in five mothers receive any postnatal check-up [11]. The percentage of rural pregnant women that received the recommended four antenatal check-ups is $21.4 \%$ as reported by the recent National Family Health Survey 2015-16 (NFHS-4) [12]. Furthermore, the coverage of these indicators vary widely across the 72 districts of Uttar Pradesh. The poor and disadvantaged section of society are the most likely to have limited access to optimal health care leading to poorer maternal and child health outcomes [13]. In rural Uttar Pradesh (UP), Rajiv Gandhi Mahila Vikas Pariyojana (RGMVP) has established a large base of volunteer-led self-help groups (SHGs), mostly constituted by poor and lower caste women, who work for poverty alleviation. To address the high burden of NMR, a health programme named "Uttar Pradesh Community Mobilization (UPCM) project" was initiated in 2011, funded by the Bill \& Melinda Gates Foundation. This was led by the Public Health Foundation of India (www.phfi.org), with a goal to reduce infant mortality by improving maternal and child health behaviour, through scaling up health intervention packages via SHG using behaviour change communication methods. The learning phase of the larger project was established across eight districts (10 blocks and 100 gram panchayats) in 2013.

In this paper, we focus on three newborn care practices: clean cord care, timely initiation of breastfeeding practices and thermal care. We (i) describe newborn care practices as reported by mother's recall and quantify the correlation of such practices within communities at block and gram panchayat (GP) level and (ii) explore the association between newborn care practices with ANC during pregnancy, number of contacts with community health workers during ANC period and place of childbirth.

\section{Methods}

This study uses baseline data of the learning phase of the abovementioned UPCM project. This survey was conducted by Population Council, Delhi, during June to August, 2013, in 15 blocks from five districts chosen purposively based on geographical diversity. These districts had the presence of self-help groups that 
concentrated on health-related activities implemented by Rajiv Gandhi Mahila Vikas Pariyojana (RGMVP), a rights-based organization that works for poverty reduction, women's empowerment and rural development in Uttar Pradesh (www.rgmvp.org/).

\section{Sampling and sample size}

GPs were smaller administrative units within these blocks. A house listing of all the SHG members was done in all the listed GPs to identify eligible women from SHG households. To identify eligible women from non-SHG households in the intervention area, a listing of non-SHG households was done in the neighbourhood of SHG households to ensure similar socio-economic characteristics of women in both the groups. The required number of non-SHG households (approximately half of the sampled SHG households) was randomly selected from each village. The eligibility criteria for inclusion of respondents in the sampling frame were (a) women of age 15 to 49 years, (b) currently married and (c) had delivered a baby in the 15 months prior to the survey. Since only one woman represented each household, we consider women nested within GPs and GPs nested within blocks. Sample size calculations were meant for the objective to measure the changes in maternal neonatal and child health indicators over time in intervention area as compared to control area [14], and hence, there are no pre hoc sample size calculations for this exploratory analysis. For this paper, we have used the overall data from all blocks for presenting the results of study objectives.

\section{Data collection}

After obtaining verbal informed consent from the mother, two sets of questionnaires, a household schedule and a woman schedule, were administered in Hindi by trained data collectors using a computer-assisted personal interview (CAPI) package designed using CSPro programme [15]. Information on the family members, socio-demography, household-level wealth and membership with any SHG were obtained. The women's questionnaire collected information on antenatal, childbirth and postnatal care practices. There were no refusals, but a $25 \%$ non-response rate was documented mostly due to the respondents being away from the village on the day of the survey or the houses of the selected respondents were found locked on repeated visits. The percentage of non-availability of respondents at the time of survey was similar across the blocks. Finally, data were collected from 2208 women of which 1709 women belonged to SHG households and 499 belonged to non-SHG households.

\section{Statistical analysis}

All analyses were performed using Stata14 (Statacorp, USA). Description of socio-demographic profile of household and mothers are presented using descriptive statistics for overall sample and categorized by SHG membership of the household. Data from mothers and their households was considered as the smallest unit, who were nested within a GP which in turn were nested within blocks. Proportion following key newborn care practices are presented along with intracluster correlation coefficient (ICC) at block level and GP level.

We computed the socio-economic score of households using principal component analysis with data of household facilities and assets at the time of interview. We categorized the score into quintiles with the first quintile being the least poor and the last quintile the poorest of the poor. We defined levels of marginalization using three indicators viz. ability to read or write, caste and socio-economic status (SES) of the household. Women belonging to scheduled caste or tribe (SC/ST) category, unable to read or write and belonging to the last two quintiles of SES were considered as the most marginalized. Least marginalized were those who had none or only one of the marginalization factors described above. The definition of newborn care practices and other variables used in the analysis is found in Table 1 . To explore the determinants of good newborn care practices, we performed multilevel random intercept logistic regression and present odds ratio (OR) with 95\% CI. We used multilevel random intercept logistic regression

Table 1 Definition of certain exposure and outcome variables

Marginalization: This consists of three components: social class, literacy and socio-economic status. Women belonging to the SC/ST class, with no ability to read and write and belonging to the last two quintiles of the socio-economic score, were defined as "most marginalized". Women with only two of the above indicators are classified as "some marginalization", and those with only one or none would fall under "least marginalized".

Access to communication: Women who had access to any one of the following: $\mathrm{TV}$, radio or newspaper, were considered to have access to means of modern communication.

SHG membership: The respondent or one of her household members is a member of SHG run by Rajiv Gandhi Mahila Vikas Pariyojana.

Antenatal visits: $0=$ who did not receive any ANC during last pregnancy, $1=1$ or 2 visits, $2=3$ and more visits either at a health facility or ANC received on Village Health and Nutrition Day (VHND) by auxiliary nurse midwife (ANM)

Home delivery: All women who gave birth at home (planned and unplanned).

Good thermal care: Those who kept baby warm by any method and those who delayed first bath of newborn beyond $48 \mathrm{~h}$

Clean cord care: Those who did not apply any foreign material on the cord.

Good breast-feeding practices: Those who initiated breast feeding within $1 \mathrm{~h}$ of birth, did not discard colostrum and did not give any pre-lacteal feeds. 
as opposed to standard logistic regression technique as the data from mothers were nested within GP and blocks leading to clustering of outcomes. This enables appropriate estimation of standard errors around the estimate. Further by the random intercept method, we allow each cluster to have its own intercept but assume the slope to be the same for all clusters. The variables to be included in the model were based on statistical significance on univariable analysis and our previous experience in this field. We also explored the interaction between marginalization and SHG membership on newborn care indicators. Figure 1 depicts the conceptual framework developed before performing the analysis.

\section{Results}

The average number of GPs per block interviewed was 8.6 (SD 1.8) (range 4-10). The number of women by GP and block along with household and mother's sociodemographic profile for the overall sample and by SHG membership is presented in Table 2. Most characteristics were similar across SHG and non-SHG households. The overall study population were predominantly Hindus, half of them belonging to SC/ST and a mean household size of 7.2. Only half of the mothers had ever attended school and only $15 \%$ of those who attended till 4th standard could read. Less than half had some access to communication, and one third of these women had their own cell phones.
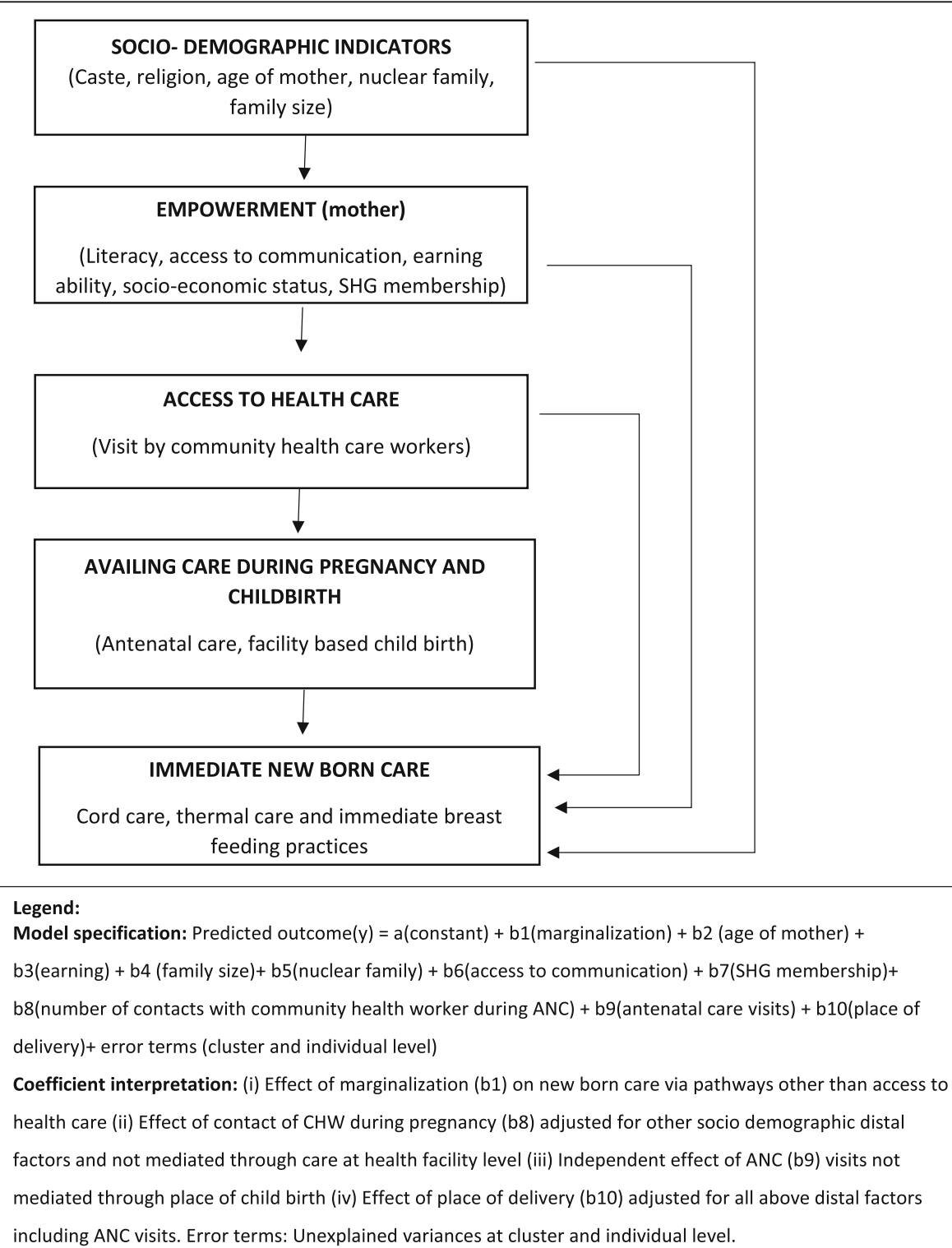

Fig. 1 Conceptual framework: determinants for newborn care by rural women 
Table 2 Household and target women characteristics

\begin{tabular}{|c|c|c|c|}
\hline \multirow[t]{2}{*}{ Household characteristics } & Overall & $\begin{array}{l}\text { Target women are SHG } \\
\text { members or belong to } \\
\text { a SHG household }\end{array}$ & $\begin{array}{l}\text { Target women not SHG } \\
\text { members or belong to } \\
\text { SHG household }\end{array}$ \\
\hline & $\begin{array}{l}N=2208 \\
G P=129 \\
\text { Block }=15\end{array}$ & $\begin{array}{l}n=1709 \\
\mathrm{GP}=129 \\
\text { Block }=15\end{array}$ & $\begin{array}{l}n=499 \\
G P=68 \\
\text { Block }=9\end{array}$ \\
\hline Number of participants per block, mean (SD) (range)/block & $\begin{array}{l}147(68) \\
(36-309)\end{array}$ & $\begin{array}{l}146(73) \\
(36-309)\end{array}$ & $\begin{array}{l}153(21) \\
(136-168)\end{array}$ \\
\hline Number participants per GP, mean (SD) (range) & $\begin{array}{l}17.1(10.8) \\
(1-67)\end{array}$ & $\begin{array}{l}17(10.9) \\
(1-67)\end{array}$ & $\begin{array}{l}16(7.6) \\
(10-29)\end{array}$ \\
\hline Hindu religion, $n(\%)$ & $2046(92.7)$ & $1586(92.3)$ & $460(92.2)$ \\
\hline Scheduled caste/tribe, $n(\%)$ & $1172(53.1)$ & $945(55.3)$ & $227(45.5)$ \\
\hline \multicolumn{4}{|l|}{ Drinking water source, $n(\%)$} \\
\hline Pump/tube well & $2013(91.2)$ & $1565(91.6)$ & $448(90.0)$ \\
\hline Open source (well/lake, etc.) & $148(6.7)$ & $111(6.5)$ & $37(7.4)$ \\
\hline Piped (house/yard) & $47(2.13)$ & $33(1.9)$ & $14(2.8)$ \\
\hline \multicolumn{4}{|l|}{ Toilet, $n(\%)$} \\
\hline Flush & $133(6.02)$ & $94(5.5)$ & $39(7.8)$ \\
\hline Pit & $43(2)$ & $30(1.8)$ & $13(2.6)$ \\
\hline Open defecation & $2032(92.03)$ & $1585(92.7)$ & 447 (89.6) \\
\hline \multicolumn{4}{|l|}{ Cooking fuel type, $n(\%)$} \\
\hline Electricity/LPG/kerosene & $59(2.7)$ & $40(2.3)$ & $19(3.8)$ \\
\hline Wood/dung/shrub & 2149 (97.3) & $1669(97.7)$ & $480(96.2)$ \\
\hline \multicolumn{4}{|l|}{ Source of lighting, $n(\%)$} \\
\hline Electricity & $572(25.9)$ & $434(25.4)$ & $138(27.7)$ \\
\hline Non-electricity & $1636(74.1)$ & $1275(74.6)$ & $361(72.3)$ \\
\hline Number of hours of power supply, mean (SD) & $7.16(2.8)$ & $7.16(2.8)$ & $7.17(2.8)$ \\
\hline \multicolumn{4}{|l|}{ Dwelling type, $n(\%)$} \\
\hline Thatched (Kuccha) & $822(37.2)$ & $653(38.2)$ & $169(33.9)$ \\
\hline Semi-concrete (semi-Pucca) & $1227(55.6)$ & $946(55.4)$ & $281(56.3)$ \\
\hline Concrete (Pucca) & $159(7.2)$ & $110(6.4)$ & $49(9.8)$ \\
\hline Household size, mean (SD) & $7.2(2.8)$ & $7.4(3.05)$ & $6.8(3.02)$ \\
\hline Nuclear type of family, $n$ (\%) & $992(44.9)$ & $739(43.2)$ & $253(50.7)$ \\
\hline \multicolumn{4}{|l|}{ Socio-economic position ${ }^{\mathrm{a}}, n(\%)$} \\
\hline 0 (least poor) & $440(20)$ & $315(18.4)$ & $125(25.1)$ \\
\hline 1 & $443(20.1)$ & $355(20.8)$ & $88(17.6)$ \\
\hline 2 (middle) & $441(20)$ & $333(19.5)$ & $108(21.6)$ \\
\hline 3 & $400(18.1)$ & $327(19.1)$ & $73(14.6)$ \\
\hline 4 (poorest) & $484(21.9)$ & $379(22.2)$ & $105(21.04)$ \\
\hline Women characteristics & $N=2208$ & $\begin{array}{l}\text { SHG households } \\
n=1709\end{array}$ & $\begin{array}{l}\text { Non-SHG } \\
n=499\end{array}$ \\
\hline Age in years, mean (SD) & $25.6(4.9)$ & $25.6(4.9)$ & $25.4(5)$ \\
\hline Ever attended school, $n(\%)$ & $1155(52.3)$ & $890(52.1)$ & $265(53.1)$ \\
\hline \multicolumn{4}{|l|}{ Those who attended till 4th, $n(\%)$} \\
\hline Cannot read or write & $888(76.1)$ & $691(76.2)$ & $197(75.8)$ \\
\hline Can read & $186(15.9)$ & $152(16.8)$ & $34(13.1)$ \\
\hline Can read and write & $93(8)$ & $64(7.1)$ & $29(11.2)$ \\
\hline
\end{tabular}


Table 2 Household and target women characteristics (Continued)

\begin{tabular}{|c|c|c|c|}
\hline Age in years started cohabiting with husband, mean (SD) & $17.4(2.3)$ & $17.4(2.3)$ & $17.5(2.3)$ \\
\hline Working (cash/kind), $n$ (\%) & $457(20.7)$ & $376(22)$ & $81(16.2)$ \\
\hline \multicolumn{4}{|l|}{ Access to communication, $n(\%)$} \\
\hline Do not read news paper & $1832(83)$ & $1430(83.7)$ & $402(80.6)$ \\
\hline Do not listen to radio & $1754(79.4)$ & $1351(79.1)$ & $403(80.8)$ \\
\hline Do not watch TV & $1384(62.6)$ & $1086(63.6)$ & $298(59.7)$ \\
\hline Access to any one of the above mode of communication & $987(44.7)$ & $756(44.2)$ & $231(46.3)$ \\
\hline \multicolumn{4}{|l|}{ Cell phone access, $n(\%)$} \\
\hline Own phone & $739(33.5)$ & $561(32.8)$ & $178(35.5)$ \\
\hline Husband phone & $948(42.9)$ & $752(44.0)$ & $196(39.3)$ \\
\hline Other's phone & $309(14)$ & $248(14.5)$ & $61(12.2)$ \\
\hline Do not use & $212(9.6)$ & $148(8.7)$ & $64(12.8)$ \\
\hline \multicolumn{4}{|l|}{ Marginalization ${ }^{\mathrm{a}}, n(\%)$} \\
\hline Least & $827(37.45)$ & $608(35.6)$ & 219 (439) \\
\hline Some & $901(40 . .8)$ & $713(41.7)$ & $188(37.7)$ \\
\hline Most & $480(21.7)$ & $388(22.7)$ & $92(18.4)$ \\
\hline Number of live births, median (IQR) & $3(1,4)$ & $3(1,4)$ & $2(1,4)$ \\
\hline Number of living children, median (IQR) & $2(1,3)$ & $2(1,3)$ & $2(1,3)$ \\
\hline At least one still birth, $n(\%)$ & $138(6.25)$ & $104(6.1)$ & $34(6.8)$ \\
\hline
\end{tabular}

${ }^{a}$ Marginalization defined as a composite of caste $(S C / S T=1$, others $=0)$, literacy (cannot read and write $=1$, only read $/$ both $=0$ ), socio-economic status $(0-4$ least poor to poorest) $(0 / 2=0,3 / 4=1)$. Total score ranges from 0 to $3.3=$ most marginalized, $2=$ some marginalization, 1 and 0 least marginalized

\section{Care during pregnancy}

Most women (84\%) had received at least one ANC visit. The mean number of ANC visits overall for the sample was 2.6 (SD 1.9). Fifty-one percent had three or more visits and $91 \%$ of the women reported at least one contact with any of the community health workers (CHWs: auxiliary nurse midwife (ANM) or Accredited Social Health Activist (ASHA) or Anganwadi worker (AWW)) during pregnancy. Thirty-two percent of the women gave birth at home. Among those who delivered at a health facility, around $45 \%$ stayed at least for $24 \mathrm{~h}$ in the facility post-childbirth. Eight percent of mothers neither received ANC nor went to a health facility for childbirth. Around three fourths of mothers reported to have not received postnatal check-up for them or their newborn. Table 3 describes the self-reported items by recall, of advice given to them regarding postnatal care in the health facility before discharge.

\section{Newborn care}

Newborn care practices (cord care, timely initiation of breast feeding and thermal care) as reported by the mothers by recall are reported in Table 4 . We found small yet significant amount of clustering of these practices due to unexplained individual- or cluster-level factors, ranging from 2 to $6 \%$ at the block level and 5 to $11 \%$ at the GP level. Only $5 \%$ of mothers reported to have followed all the mentioned newborn practices.
One fourth of mothers practised clean cord care. Ghee was the commonest material applied (33\%), followed by ash (13\%) and talcum powder (12\%). Application of gentian violet on the cord was $4.6 \%$ and was higher among those who had at least one ANC check-up than those who did not receive any ANC check-up and also higher among those who gave birth at home (6\%) than those who delivered at a health facility (1.7\%). Mothers who were earning members of the family, SHG members and those who had at least one ANC check-up were less likely to follow clean cord care when compared to nonearning mothers, non-SHG members and those who did not have even one ANC check-up. However, giving birth at a hospital had a positive association with clean cord care (Table 5). Only 35\% of mothers covered the newborn immediately after birth and delayed the first bath of the baby by $48 \mathrm{~h}$. Place of birth was strongly and positively associated with good thermal care, and access to communication showed some positive association with thermal care. Receiving ANC and delivering at a facility increased the likelihood of following appropriate breastfeeding practices. Further, appropriate breast-feeding practices were less likely followed by women who were least marginalized than the most marginalized women; however, this association was not statistically significant (Table 5). The effects of marginalization were similar across SHG and non-SHG households (not shown in table). Women with SHG membership or with SHG 
Table 3 Information on last live childbirth as reported by recall of mother

\begin{tabular}{ll}
\hline Details last pregnancy and childbirth & $N=2208$ \\
\hline $\begin{array}{l}\text { Received at least one ANC check-up last pregnancy, } \\
n \text { (\%) }\end{array}$ & $1842(83.4)$ \\
Number of ANC visits, mean (SD) & $2.5(1.9)$ \\
Women with at least four ANC, $n$ (\%) & $462(20.9)$ \\
Had contact with any of the community health & $2007(90.9)$ \\
workers (CHWs) during ANC, $n$ (\%) & \\
$\begin{array}{l}\text { Number of contacts with CHWs during ANC, } \\
\text { median (IQR) }\end{array}$ & $5(3,7)$ \\
Place of childbirth (\%) & \\
$\quad$ Home & $701(31.8)$ \\
$\quad$ Health centre & $937(42.4)$ \\
$\quad$ Hospital & $570(25.8)$ \\
Planned place of childbirth, $n$ (\%) & $1682(76.2)$ \\
$\begin{array}{l}\text { Vital status of offspring at time of interview } \\
\text { (alive), } n \text { (\%) }\end{array}$ & $2189(99.1)$ \\
Male child, $n$ (\%) & $1115(50.5)$ \\
Normal delivery, $n$ (\%) & $1349(90.2)$ \\
Instrumental, $n$ (\%) & $65(4.4)$ \\
LSCS, $n$ (\%) & $81(5.4)$
\end{tabular}

Duration of stay in days in the hospital/health $\quad 0.62(0.12,2)$ centre, median

(IQR) stayed at least for $24 \mathrm{~h}$ (1492), $n$ (\%)

Received advice was given before discharge (1495), n (\%)

Initiation of breast feeding within $1 \mathrm{~h}$

Feeding colostrum

$1176(78.7)$

Exclusive breast feeding

$1213(81.1)$

Kangaroo mother care

Bathing after $24 \mathrm{~h}$

Information on danger signs

Mother

Newborn

Contraception due to breast feeding

Postnatal care, $n(\%)$

None after childbirth

Within 42 days but after 7 days of childbirth

Within 7 days of childbirth

Number of visits made $(n=580)$, median (IQR)

Median time in days of first PNC visit $(n=580)$, median (IQR)

Home visit by community health worker after childbirth, $n(\%)$

Within 42 days

$1078(48.8)$

After 42 days

$222(10.1)$

$908(41.1)$

$2(1,4)$ members within the household were independently associated with newborn care practices, but the direction of association for each of the outcome was inconsistent. The density of SHG households within a GP also did not have any effect on the newborn care practices (not shown in table). There was no significant association between number of contacts with community health workers and newborn care.

Mother's awareness (at the time of interview) of newborn care was assessed. Of the 2208 mothers, around three fourths of the mothers were aware of giving birth in a health facility as a safe practice and $67 \%$ mentioned that they would take the newborn to a health facility after 1 week of birth. Only 58\% were aware about initiating breast milk within $1 \mathrm{~h}$ of birth. Also, 9\% were aware of clean cord care practice, and only 3\% knew about Kangaroo mother care (KMC).

\section{Discussion}

Our results show that institutional delivery had a significant impact on newborn care practices in the context of rural UP. Number of ANC visits was associated with breast-feeding practices in a positive way but not with clean cord care practices. The presence of an SHG member in the household, density of SHG within GP and contact with CHWs during ANC did not seem to be associated with newborn care in this population.

The focus of maternal and child health programmes have been in delivering ANC and promoting institutional delivery with the aim of reducing maternal and neonatal deaths. A population survey from Bihar showed significant relationship between newborn care and neonatal mortality [3] with odds of neonatal death increasing 3.5 times when delayed bathing practices was not followed and 2.5 times with failure to practise Kangaroo mother care. Our cross-sectional study across the rural villages of five districts of Uttar Pradesh has shown that only $5 \%$ of mothers reported to have practised all six newborn care practices correctly (clean cord care, delayed bathing $(>48 \mathrm{~h})$, thermal care, timely initiation of breast feeding (within $1 \mathrm{~h}$ ), not discarding colostrum and not feeding pre-lacteal feeds). There was also variation of newborn care practices between communities, and it should be taken into consideration while implementing any intervention to optimize benefits.

In our study, antenatal care was found to be independently associated with correct breast-feeding practices and thermal care but not with clean cord care. A study from Nigeria showed that cord care education given to mothers at antenatal clinics during ANC was not associated with actual cord care and the information provided also was not standardized [16]. This same study data showed that clean cord care practice was associated with mothers' education, gender of child (favouring males) 


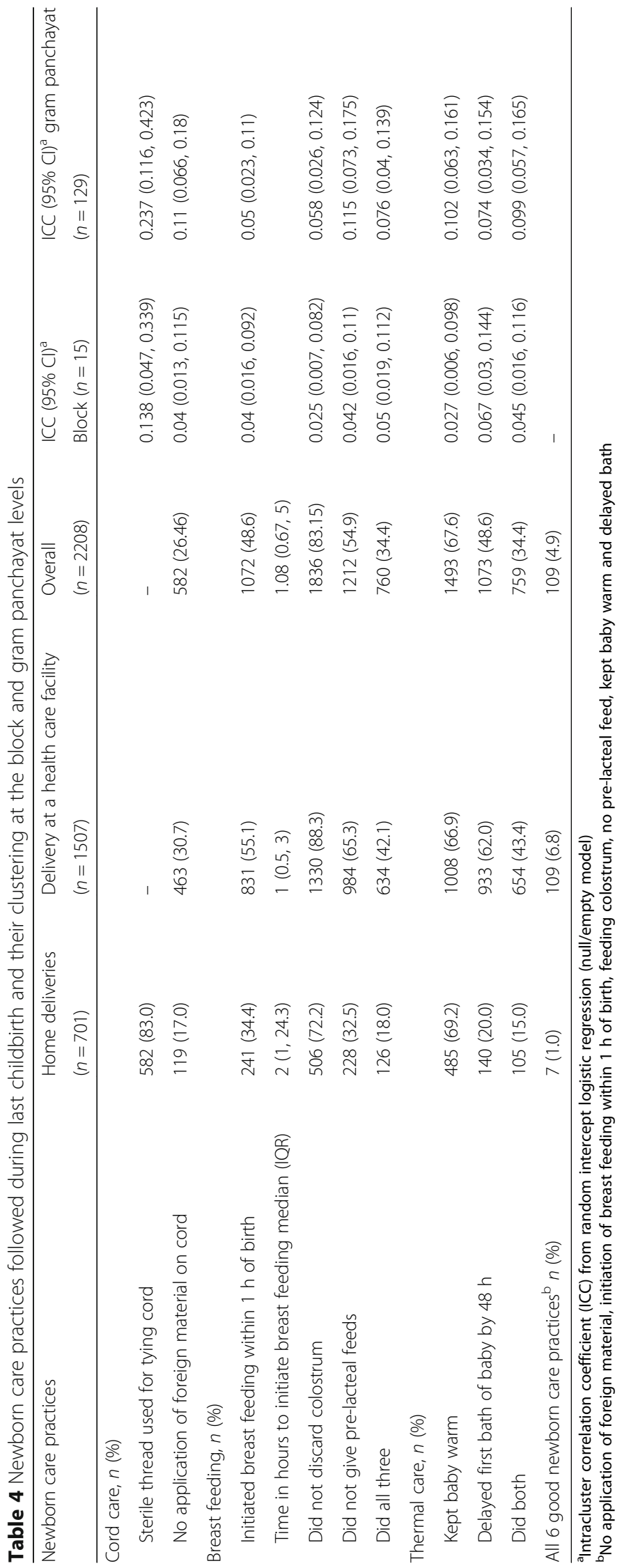




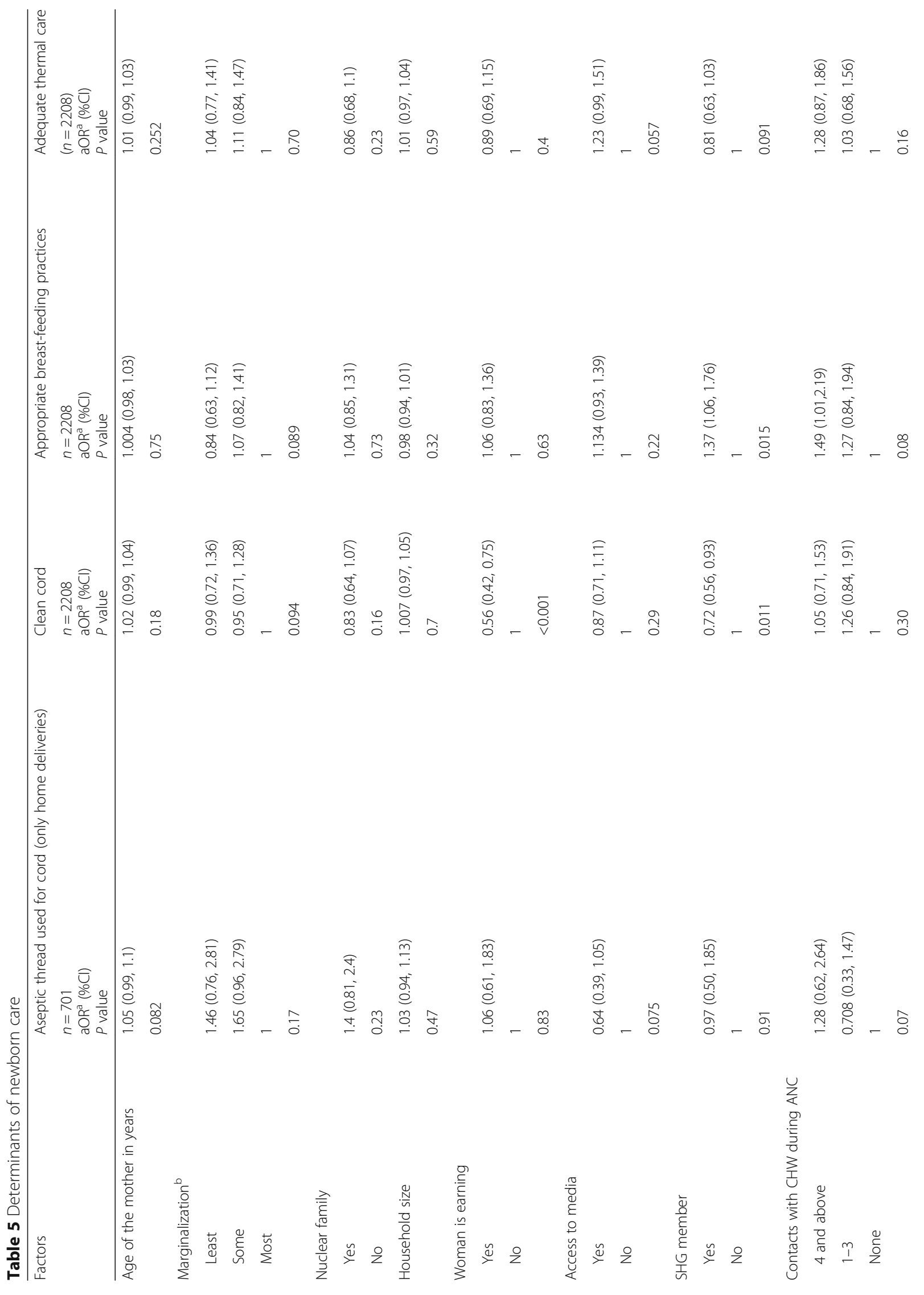


Devasenapathy et al. Journal of Health, Population and Nutrition (2017) 36:30

Page 10 of 12

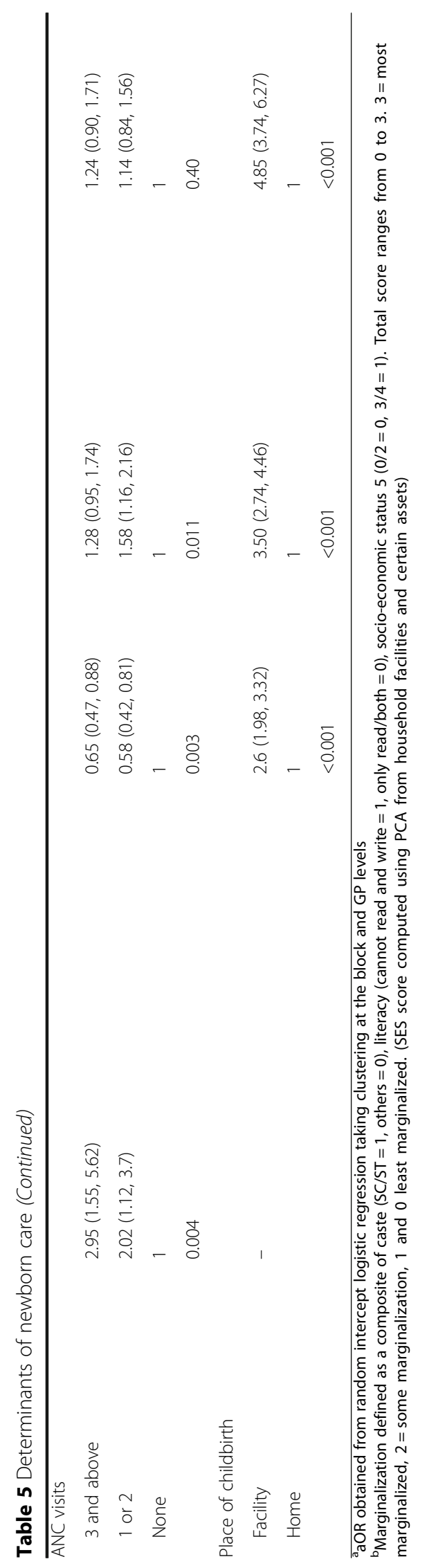


and teaching hospital [17]. Further studies are required in states with a high burden of neonatal mortality and morbidity to assess the association of quality of ANC with cord care practices. The positive association of facility-based childbirth and newborn care practices that we demonstrate here is concordant to another study done in rural UP [8].

While there is a definitive evidence of high risk of still birth and neonatal deaths with poor socio-economic status, we did not find any association of newborn care practices with level of marginalization. This could be because newborn care is more influenced by tradition and culture and community practices rather than the socioeconomic status, especially in situations like ours where nearly one third of the deliveries took place at home. This underscores the importance of the spread of appropriate messages across all strata of society rather than focusing only on the poorest.

Thermal care did not seem to have an association with the number of ANC visits irrespective of the place of childbirth. This indicates that more than the number of ANCs, it is the quality of ANCs which matter. It is also suggested that four antenatal visits may not impact the outcome of pregnancy till the coverage is $60 \%$ [2]. In our survey, only $20 \%$ of women had four or more ANC visits, which is far less than what is required.

Community health workers play a major role in imparting knowledge related to $\mathrm{MNCH}$ care during antenatal and postnatal period that can impact health outcomes [18, 19], and there is a compelling evidence from developing nations about the effectiveness of community health workers in improving maternal and child health [20] and specifically in improving newborn care practices. However, in our analysis, the number of community health worker visits to households during pregnancy did not have any effect on the newborn care practices. This could be due to the quality of the information exchange that happens during contact sessions. ASHA and other community link workers receive incentives for antenatal visits and delivery, but there are no incentives for delivering health promotion at the homes of these mothers. Our survey showed that around $40 \%$ of mothers were not visited by CHWs in the postnatal period. There is a need for improving the quality of training, supportive supervision and adequate follow-up. Given that the time spent by mothers after childbirth in facilities is not more than $24 \mathrm{~h}$ and the inability to devote quality time by hospital staff due to excess workload, this gap should be compensated by ensuring regular home visit by a community health worker to sensitize mothers regarding newborn care especially during the immediate postnatal period. The presence of CHWs is a huge opportunity existing in communities. Efforts are underway to train them in order to promote newborn care practices. However, there is a lack of monitoring and accountability [10]. The lack of an apparent impact could be because of the fact that existing CHWs were not fully trained when the data were collected.

One of the key limitations of this study is the length of recall period (15 months) of newborn care practices and antenatal care during pregnancy. The recalled practices could have been influenced by the other information the mother would have gained before the survey. Hence, the percentage of respondents reporting good newborn care practices could be an overestimate as shown by the discrepancy between the awareness of clean cord care (9\%) and reported clean cord care practice (25\%). We did not collect information at the GP or block level which could have allowed us to explore reasons for cluster-level factors affecting newborn care practices. Despite its limitations, this data provides insights into the actual practices on a large sample of population in one of the states with poor health indicators. Locally collected data would help frame an intervention package that would have greater acceptability by the population.

In this sample of population, an inconsistent association was found between SHG membership and newborn care practices. At the time of the survey, SHGs were not utilized for improving awareness about healthy mother and child practices and this could explain the lack of this association. However, there is evidence that self-help groups can have an impact on improving health care utilization and practices [21, 22]. In our setting, the SHG platform could be a potential resource to improve community behaviours and may provide a more sustainable solution to a problem that the state is grappling with.

\section{Conclusion}

Based on the survey findings, we can conclude that there is a need for improving newborn care among the rural population living in Uttar Pradesh. This would impact on reduction of neonatal morbidity and mortality. While the thrust by the existing government programmes for the promotion of at least four antenatal care visits and institutional deliveries should continue, it is time that the quality of care offered in the facilities and the counselling given by community health workers both during antenatal and the immediate postnatal period be stepped up. Mobilizing selfhelp groups and strengthening women's linkages with community health workers may be promising, and the evaluation of this community mobilization project could provide some evidence in the context of rural UP. 


\section{Abbreviations}

ANC: Antenatal care; CHWs: Community health workers; GP: Gram panchayat; RGMVP: Rajiv Gandhi Mahila Vikas Pariyojana; SES: Socio-economic status; SHGs: Self-help groups; UP: Uttar Pradesh

\section{Acknowledgements}

This publication used secondary analysis based on data collected by the Population Council for the Bill \& Melinda Gates Foundation (BMGF)-funded "Uttar Pradesh Community Mobilization" consortium project. The consortium consisted of Public Health Foundation of India (PHFI), Rajiv Gandhi Mahila Vikas Pariyojana (RGMVP), Population Council (PC) and Boston University (BU)

\section{Funding}

This project is funded by the Bill \& Melinda Gates Foundation (grant number OPPI033910) awarded to the Public Health Foundation of India. The funding agency had no role in the design and conduct of the study. The funding agency had no role in the analysis, interpretation of the results and writing of this manuscript

\section{Availability of data and materials}

The data supporting the findings of this study is available with the corresponding author (ND) and the data can be shared at request to the corresponding author (ND).

\section{Authors' contributions}

ND analysed the data, interpreted the results and wrote the first draft of the manuscript. DA and SS gave inputs in the conceptualization of the paper. $\mathrm{AH}$ reviewed the various versions of the manuscript and made substantial contribution in strengthening the content of the methodology and analysis. DM and SN reviewed and provided inputs in improving the manuscript. DA, SS, JA and NM reviewed the manuscript draft and provided inputs. All authors read and approved the final manuscript.

\section{Competing interests}

The authors declare that they have no competing interests.

\section{Consent for publication}

Not applicable.

\section{Ethics approval and consent to participate}

This study protocol was reviewed and approved by the Institutional Review Board of the Population Council, New York. Informed consent in the local language for participation in the study was obtained from each participant by trained interviewers before the start of the interviews.

\section{Publisher's Note}

Springer Nature remains neutral with regard to jurisdictional claims in published maps and institutional affiliations.

\section{Author details \\ ${ }^{1}$ Indian Institute of Public Health-Delhi, Plot No. 47, Sector 44, Institutional Area, Gurgaon 122002, India. ${ }^{2}$ Uttar Pradesh Community Mobilization Project, Public Health Foundation of India, Gurgaon 122002, India. ${ }^{3}$ Population Council, Zone 5A, Ground Flr, India Habitat Centre,Lodhi Road, New Delhi 110003, Delhi, India. ${ }^{4}$ Rajiv Gandhi Mahila Vikas Pariyojana (RGMVP), Kanpur Road, Rana nagar, Raebareli (UP)-229001, India. ${ }^{5}$ Indian Institute of Public Health Gandhinagar, Opp. Air Force Headquarters, Palej Road, Sector 30, Gandhinagar, Gujarat, India.}

Received: 22 November 2016 Accepted: 8 June 2017

Published online: 21 June 2017

\section{References}

1. Child mortality statistics: available at http://data.unicef.org/countries/IND. html, Accessed on 24th May, 2016

2. McClure EM, Pasha O, Goudar SS, Chomba E, Garces A, Tshefu A, et al. Epidemiology of stillbirth in low-middle income countries: a Global Network Study. Acta Obstet Gynecol Scand. 2011;90(12):1379-85.
3. Kumar GA, Dandona R, Chaman P, Singh P, Dandona L. A population-based study of neonatal mortality and maternal care utilization in the Indian state of Bihar. BMC Pregnancy Childbirth. 2014;14:357.

4. WHO recommendations on"Postnatal care of the mother and newborn", 2013. Available at http://apps.who.int/iris/bitstream/10665/97603/1/ 9789241506649_eng.pdf. Accessed on 24th May, 2016.

5. Hollowell J, Kurinczuk JJ, Oakley L, Brocklehurst P, Gray R. A systematic review of the effectiveness of antenatal care programmes to reduce infant mortality and its major causes in socially disadvantaged and vulnerable women. Oxford: National perinatal Epidemiology Unit; 2009.

6. Mangwi Ayiasi R, Kasasa S, Criel B, Garimoi Orach C, Kolsteren P. Is antenatal care preparing mothers to care for their newborns? A community-based cross-sectional study among lactating women in Masindi, Uganda. BMC Pregnancy Childbirth. 2014;14:114.

7. Fadel SA, Ram U, Morris SK, Begum R, Shet A, Jotkar R, et al. Facility delivery, postnatal care and neonatal deaths in India: nationally-representative casecontrol studies. PLoS One. 2015:10(10):e0140448.

8. Khan ME, Hazra A, Bhatnagar I. Impact of Janani Suraksha Yojana on selected family health behaviors in rural Uttar Pradesh. J Fam Welf, Special Issue. 2010:56:9-22.

9. Estimates of mortality indicators: http://www.censusindia.gov.in/vital_ statistics/SRS_Report_2012/11_Chap_4_2012.pdf, accessed on 22nd June, 2016

10. State of India's newborn 2014: http://www.newbornwhocc.org/SOIN PRINTED\%2014-9-2014.pdf, accessed on 22nd June, 2016.

11. Annual health survey 2102-2013 factsheet. http://www.censusindia.gov.in/ vital_statistics/AHSBulletins/AHS_Factsheets_2012-13/FACTSHEET-UTTAR_ PRADESH.pdf, accessed on 22nd June, 2016.

12. National health family survey-4, 2015-16 Fact sheet Uttar Pradesh. International Institute of Population Sciences. http://rchiips.org/NFHS/pdf/ NFHS4/UP_FactSheet.pdf. Accessed on 23rd May 2017.

13. Sanneving L, Trygg N, Saxena D, Mavalankar D, Thomsen S. Inequity in India: the case of maternal and reproductive health. Glob Health Action. 2013;6:19145.

14. Khan ME, Agrawal PK, Hazra A, Dixit A, Bhatnagar I, Ahmad J, Ahmad D. 2014. Maternal and newborn health behaviors in rural Uttar Pradesh: findings from learning phase baseline survey, 2013. New Delhi: Population Council. http://www.popcouncil.org/uploads/pdfs/2014RH_ MaternalNewbornHealthUP.pdf. Accessed on 5th June, 2016.

15. Creating the "MyCAPI_Intro" data entry application. https://www.census. gov/population/.../files/cspro/CSPro-CAPI-Getting\%20Started.pdf. Accessed on 26th June, 2016.

16. Abhulimhen-lyoha $\mathrm{BI}$, Ibadin $\mathrm{MO}$. Cord care education and its content given to mothers at antenatal clinics in various health facilities in Edo state, Nigeria. Sahel Med J. 2015;18:129-33.

17. Abhulimhen-lyoha BI, Ibadin MO. Determinants of cord care practices among mothers in Benin City, Edo State, Nigeria. Niger J Clin Pract. 2012; 15(2):210-3.

18. Lassi ZS, Das JK, Salam RA, Bhutta ZA. Evidence from community leve inputs to improve quality of care for maternal and newborn health: interventions and findings. Reprod Health. 2014;11 Suppl 2:S2.

19. Bang AT, Bang RA, Baitule SB, Reddy MH, Deshmukh MD. Effect of homebased neonatal care and management of sepsis on neonatal mortality: field trial in rural India. Lancet. 1999;354(9194):1955-61.

20. Aboubaker S, Qazi S, Wolfheim C, Oyegoke A, Bahl R. Community health workers: a crucial role in newborn health care and survival. J Glob Health. 2014:4(2):020302

21. Saha S, Annear PL, Pathak S. The effect of Self-Help Groups on access to maternal health services: evidence from rural India. Int J Equity Health. 2013; 12:36.

22. Tripathy $P$, Nair N, Barnett S, Mahapatra R, Borghi J, Rath S, et al. Effect of a participatory intervention with women's groups on birth outcomes and maternal depression in Jharkhand and Orissa, India: a cluster-randomised controlled trial. Lancet. 2010:375(9721):1182-92. 